\title{
Das Oberösterreichische Landesarchiv. Spuren von Dichtern, Literatur und Kultur
}

Was stellt man sich gemeinhin unter einem Landesarchiv vor? Vermutlich eine altehrwürdige Einrichtung, in der sich Regalmeter um Regalmeter Akten aneinanderreihen, mit einer mehr oder weniger dicken Staubschicht überzogen. Wie bei jedem Klischee findet sich auch hier ein Funken Wahrheit. Das Oberösterreichische Landesarchiv (OÖLA) kann tatsächlich auf eine über 120-jährige Geschichte zurückblicken, die Regale sind mit über 35 Laufkilometern an Archivalien gefüllt, und der Staub wird kontinuierlich von fleißigen MitarbeiterInnen in die Schranken gewiesen, da Staub und Archivalien aus konservatorischer Sicht keine gut Kombination bilden. Aber das ist nur ein ganz kleiner Blick auf das Wesen eines Landesarchivs. An die Laufkilometer schließen sich inzwischen bereits Terabytes an digitalen Archivalien an, und die Anforderungen an die ArchivarInnen steigen kontinuierlich. Heute stellt sich nicht mehr die Frage, ob eine Archivierung unserer digitalen Gegenwart möglich ist - sie ist es -, die Frage ist eher, mit welchem personellen und finanziellen Aufwand sie verbunden ist.

Die Anfänge des OÖLA reichen bis ins 19. Jahrhundert zurück. Am 10. Jänner 1896 genehmigte der oberösterreichische Landtag das provisorische Organisations-Statut für das OÖLA und bewilligte Gelder zur Adaptierung von Räumlichkeiten. Unterstellt wurde es direkt dem Landesausschuss. Die Ausgangsbasis bildete das alte Archiv der vier Landstände ob der Enns. Der Zusammenbruch der österreichisch-ungarischen Monarchie wirkte sich auch auf die Verwaltung und damit auf das Archiv aus. Im Dezember 1919 beschloss die oberösterreichische Landesregierung, dass alle älteren Schriftbestände der staatlichen Verwaltung bis zum Jahr 1850 als „Archiv der Landesregierung“ an das Landesarchiv übergeben werden sollten. Das OÖLA vollzog damit endgültig den Schritt zu einem Zentralarchiv des Landes (vgl. Haider 1996). Trotz eines Neubaus Ende der 1960erJahre sind die Platzreserven erschöpft und es mussten Bestände ausgelagert werden. Derzeit verfügt das Landesarchiv über drei Außendepots. Im adaptierten Brecher Buchleiten in Ampflwang befinden sich Bestände zum Schwerpunkt Bergbau, im Stift Lambach die sog. Neuen Grundbücher und in Schloss Hartheim (Gemeinde Alkoven) Krankenhausbestände.

Ein Meilenstein in der Geschichte des OÖLA war das Inkrafttreten des „Landesgesetzes über die Sicherung, die Aufbewahrung und die Nutzung von öffentlichem Archivgut sowie die Tätigkeit der damit betrauten Archive“ (LGBl. Nr. 83/2003), kurz „Ö̈. Archivgesetz“, im Juli 2003. Der Begriff „Archivieren“ wird darin folgendermaßen definiert: „[E]ine Tätigkeit im öffentlichen Interesse, die 
das Erfassen, Bewerten, Übernehmen, dauernde Verwahren oder Speichern sowie das Erhalten, Restaurieren, Ordnen, Erschließen und Nutzbarmachen von Archivgut umfasst“ (LGBl. Nr. 83/2003, § 2 Z. 3). Das „Ö̈. Archivgesetz“ regelt u. a., wer seine Unterlagen dem OÖLA zur Übernahme anbieten muss, dazu gehören beispielsweise alle Behörden und Dienststellen des Landes Oberösterreich, die Landesregierung und der Landtag, und wer selbst verpflichtet ist, zu archivieren. ${ }^{1}$

Archivieren ist kein Selbstzweck. Der Erhalt von Archivgut über die Jahrhunderte hat nur einen Wert, wenn dieses auch dazu genutzt wird, Wissen und Informationen zu gewinnen. Die Gruppe der BenutzerInnen des OÖLA hat sich seit der Gründung massiv verändert. Sieht man alte „Benutzerreverse“ aus den 1920er- und 1930er-Jahren durch, dominiert die universitäre Wissenschaft. In den letzten Jahrzehnten ist es gelungen, die Hemmschwelle für einen Archivbesuch deutlich zu senken. Neben StudentInnen, die an ihren Abschlussarbeiten schreiben, forschen vornehmlich an Geschichte Interessierte zu ihren Familien oder zur Vergangenheit ihrer Heimatorte. Dazu kommt ein großer Kreis an BenutzerInnen mit Rechtsfragen, die in den Grundbüchern, Grundbuchanlegungsakten und den Grundbuchurkunden recherchieren. Eine der größten Nutzergruppen macht naturgemäß die Landesverwaltung selbst aus. Nur ein Bruchteil dieser BenutzerInnen ist auch tatsächlich im Lesesaal anwesend. Familienforschung kann beispielsweise bereits über einschlägige Internetportale betrieben werden, da die dafür notwendige Hauptquellen, die Pfarrmatriken, bereits digitalisiert wurden und im Netz zur Verfügung stehen. Dasselbe gilt auch für historische Ausgaben oberösterreichischer Zeitungen oder Urkundenbestände.

Auch wenn die Bestände der oberösterreichischen Landesverwaltung einen großen Teil des Archivgutes des OÖLA ausmachen, ist dieses kein reines Verwaltungsarchiv. Es umfasst bspw. auch Unterlagen aus Gerichten, aufgelassenen Stiften, Vereinen, Gemeinden, Krankenhäusern sowie Herrschaften. Dazu kommen Sammlungen und Nachlässe unterschiedlichster Herkunft. ${ }^{2}$ Sammlungen machen jedoch nur einen geringen Teil eines Landesarchives aus. Ohne sich in den Feinheiten der Diskussion um die Frage von Bestandsdefinitionen zu ver-

1 Eine große Gefahr, mit der Archive immer wieder konfrontiert sind, ist die Tatsache, dass es sich bei potenziellem Archivgut in der überwiegenden Mehrheit der Fälle um Unikate handelt, einmal verloren, sind sie nicht wieder zu beschaffen. Kein Archiv der Welt ist vor Verlusten gefeit, die durch mangelndes Wissen über Archivierung entstehen. Allzu viel historisch Relevantes hat bereits unrettbar in Papiermühlen sein Ende gefunden. Umso wichtiger war es, eine gesetzliche Grundlage für Archivierung zu schaffen, die nicht zuletzt den öffentlichen Archiven in ihren Organisationen ein Mehr an Aufmerksamkeit gebracht hat.

2 Die Bestandsübersicht des OÖLA online unter: www.landesarchiv-ooe.at. 
lieren, lässt sich sehr vereinfacht sagen, dass nicht zu einem Thema gesammelt, sondern archivwürdiges Schriftgut eines Produzenten als Bestand übernommen und bearbeitet wird. So würde man beispielsweise nicht die Akten des AdalbertStifter-Institutes/StifterHauses nach Themen wie „Ausstellungen“, „Publikationen“, „Organisation“ aufteilen und mit den Akten des Oberösterreichischen Landesmuseums $\mathrm{zu}$ Ausstellungen, Publikationen und Organisation mischen, sondern einen Bestand „Stifter-Institut“ bilden und einen Bestand „Landesmuseum“.

Ausnahmen bestätigen bekanntlich die Regel, und auch im OÖLA gibt es diese Ausnahmen, beispielsweise den Bestand „Stifterakten“. Da Adalbert Stifter bei einer Behörde arbeitete, finden sich die Spuren seiner beruflichen Tätigkeit im Aktenbestand der k.k. Statthalterei im Bereich Unterricht. Entgegen dem oben ausgeführten Provenienzprinzip, nämlich Unterlagen in ihrem Entstehungszusammenhang zu belassen, wurden diese „Stifterakten“ aus dem Bestand gezogen, nicht zuletzt um sie besser schützen zu können. Die Unterschrift Stifters unter einem Akt weckt schneller Begehrlichkeiten als die Unterschrift eines längst vergessenen Sachbearbeiters. An den Bestand „Stifterakten“ angeschlossen wurden in der Folge Einzelstücke wie beispielsweise die Verlassenschaftsabhandlungen von Adalbert Stifter und seiner Frau Amalia. Was für Stifter-ForscherInnen eine Erleichterung bedeutet, erschwert hingegen die Arbeit von WissenschaftlerInnen, die sich mit Schulfragen im Land ob der Enns im 19. Jahrhundert beschäftigen und möglicherweise genau jene Akten suchen, die zufällig Stifter bearbeitet hat. Mit einer detaillierten Dokumentation und Verzeichnung der aus einem Bestand gezogenen Einzelakten lässt sich diese Problematik allerdings gut lösen.

Im Folgenden sollen anhand von einigen Beispielen Spuren von Dichtern, Literatur und Kultur im OÖLA vorgestellt werden.

\section{Geburt und Tod}

Jede und jeder von uns findet sich alleine schon durch ihre bzw. seine Geburt in einem Archiv wieder: Ursprünglich führten die anerkannten Kirchen im staatlichen Auftrag die Personenstandsbücher zur Beurkundung von Geburt, Eheschließung und Tod. Nach dem „Anschluss“ Österreichs an das Deutsche Reich änderte sich dies und das Personenstandswesen ist bis heute eine Aufgabe der Gemeinden im übertragenen Wirkungsbereich (vgl. Personenstandsgesetz 2013 BGBl. I Nr. 16/2013). Das OÖLA erhielt im Jahr 1975 vom Bischöflichen Ordinariat Linz die Abschriften der Pfarrmatriken aller oberösterreichischer Pfarrgemeinden von 1819 bis 1940. Die Originale befinden sich in den Pfarren. 


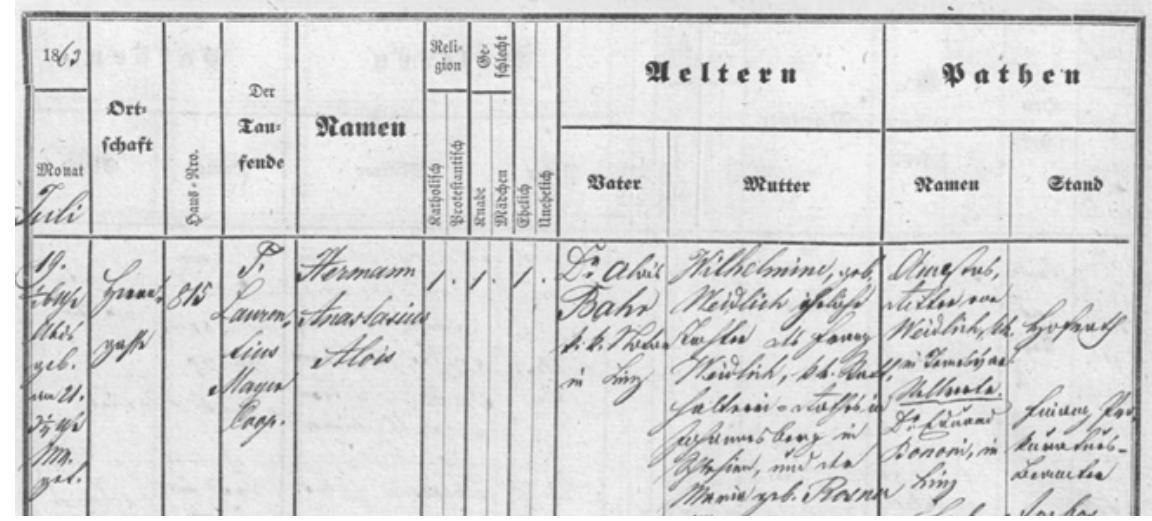

Abb. 1: Taufeintrag zu Hermann Bahr.

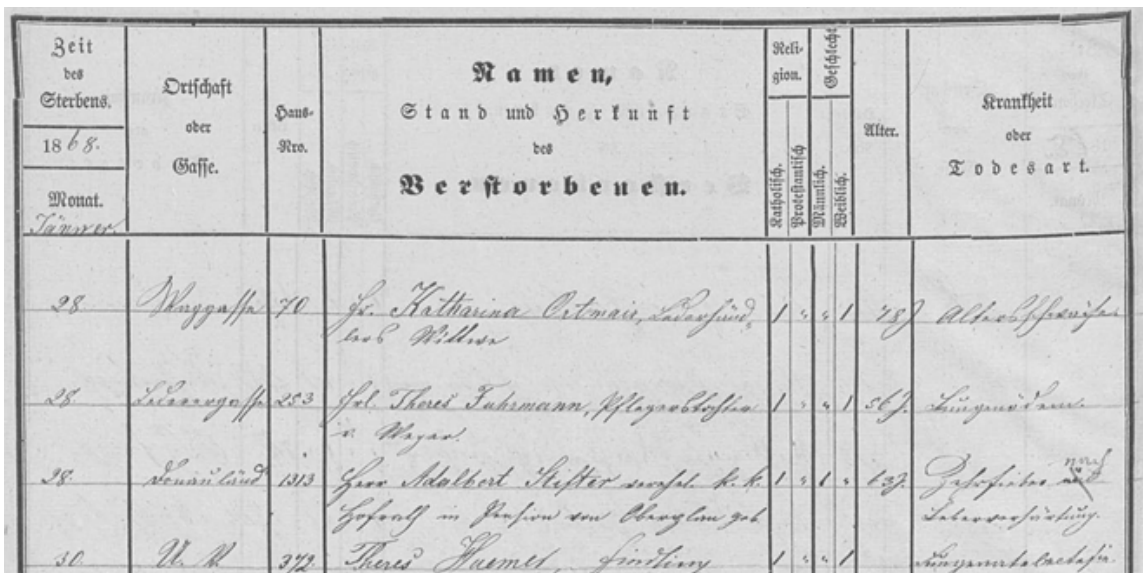

Abb. 2: Sterbeeintrag zu Adalbert Stifter.

Aus einem Taufeintrag lassen sich eine Reihe von Informationen über den Täufling herauslesen. Wann und wo er geboren und getauft wurde, wer seine Eltern und Paten waren und welchen Beruf sie ausübten. Im Taufbuch der Pfarre Linz-St. Matthias ist beispielsweise im Juli 1863 für den 19. die Geburt von Hermann Anastasius Alois in der Herrengasse Nr. 815 verzeichnet, Sohn des k.k. Notars Dr. Alois Bahr und seiner Frau Wilhelmine, Tochter des k.k. StatthaltereiRathes Franz Weidlich und seiner Frau Maria. Die Taufe fand zwei Tage später statt. 


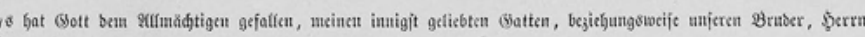 \\ Mtomlhert Stifter,}

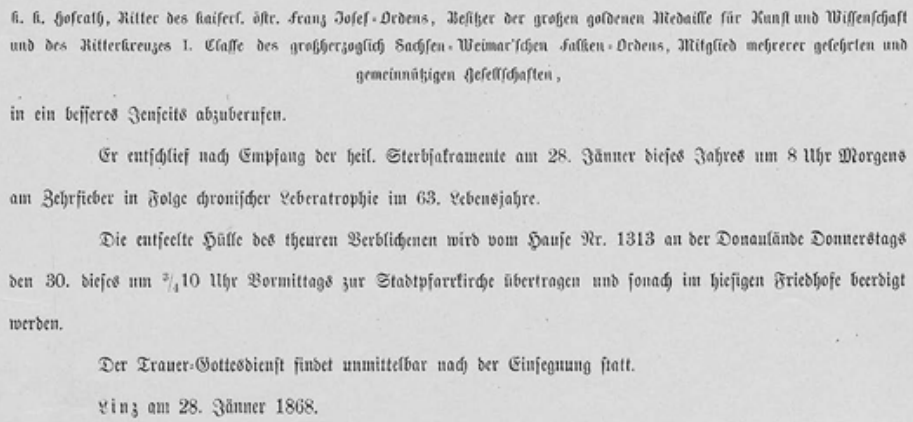

Abb. 3: Partezettel Adalbert Stifters.

Im Sterbebuch der Stadtpfarre Linz findet sich am 28. Jänner 1868 der Eintrag zum Tod von Adalbert Stifter ,verehel. k.k. Hofrath in Pension von Oberplan geb.“; als Todesursache wurde „Zehrfieber nach Leberverhärtung“ genannt.

Noch detailliertere Informationen $\mathrm{zu}$ Verstorbenen bieten Partezettel. Die Partezettelsammlung des OÖLA entstand aus der Zusammenführung zweier unterschiedlicher Sammlungen 1983. Eine alphabetisch geführte Amtssammlung wurde mit der „Sammlung Lindner“ vereinigt, in der sich vor allem Parten von Adeligen und Persönlichkeiten des öffentlichen und kulturellen Lebens aus den Jahren 1824/25 befanden.

Stifters Tod wurde von seiner Gattin Amalia, geborene Mohaupt, seinen Brüdern Anton Stifter (Lederhändler), Martin Stifter (Hausbesitzer), Johann Stifter (Hausbesitzer), Jakob Mayer (Oberingenieur) und seiner Schwester Anna Stifter, verheiratete Schopper, bekannt gegeben. Sein Titel „k.k. Hofrath“ und seine Auszeichnungen wie das „Ritterkreuz I. Classe des großherzoglich SachsenWeimar'schen Falken-Ordens“" wurden genannt, sowie Tag und Stunde des Todes und Tag und Ablauf der Beerdigung. Von seinem Wohn- und Sterbeort, Donaulände Nr. 1313, brachte man Stifters Sarg in die nahegelegene Stadtpfarrkirche, 


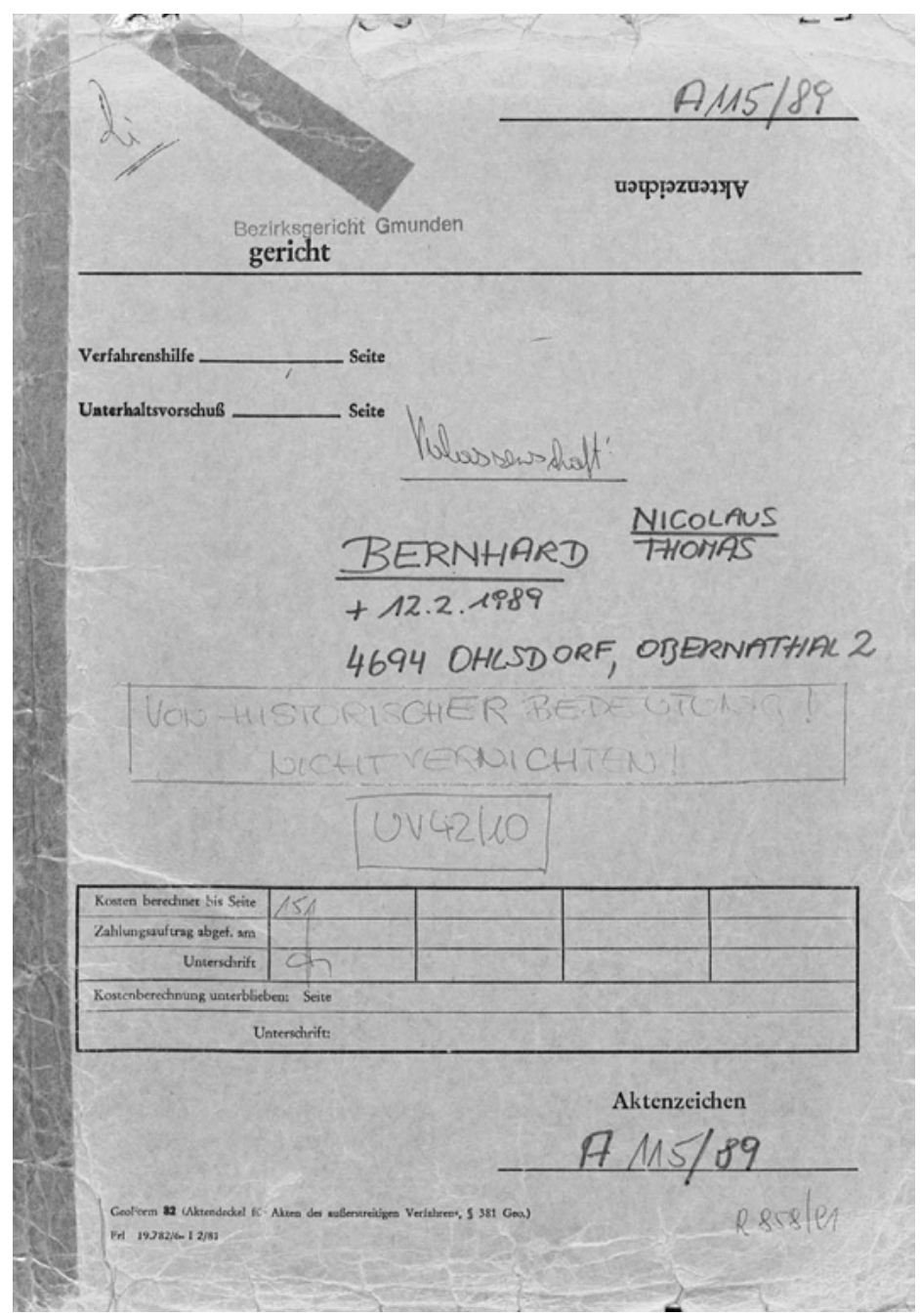

Abb. 4: Verlassenschaftakt Thomas Bernhard.

die Beerdigung fand am Barbarafriedhof statt. Der Trauer-Gottesdienst wurde unmittelbar nach der Einsegnung abgehalten.

Mit dem Tod eines Menschen wird in Österreich automatisch ein Verlassenschaftsverfahren beim zuständigen Bezirksgericht eröffnet, unabhängig davon, ob ein Vermögen vorhanden ist oder nicht. In den dazugehörigen Akten finden sich Informationen zum Verstorbenen, seiner Familie und seinen Erben, sowie zu seinem Vermögen. 
Auf dem Abhandlungsakt des Schriftstellers Thomas Bernhard befindet sich auch der in $\S 382$ der Geschäftsordnung für die Gerichte I. und II. Instanz vorgesehene Vermerk ,von historischer Bedeutung. Nicht vernichten“. Dieser ist auf richterlicher Anordnung anzubringen,

wenn der Akt wegen seines Inhaltes oder wegen der beteiligten Personen von geschichtlicher oder kultureller Bedeutung für die Erforschung und das Verständnis der Geschichte und Gegenwart in politischer, wirtschaftlicher, sozialer oder kultureller Hinsicht sowie bezüglich Gesetzgebung, Rechtsprechung, Verwaltung und den Schutz allgemeiner oder besonderer bürgerlicher Rechte ist [...]. (BGBl. Nr. 264/1951)

\section{Besitz}

Wer in Österreich im Besitz von Grund und Boden ist, hinterlässt seine Spuren automatisch im Grundbuch und den mit ihm verbundenen Unterlagen wie den Grundbuchurkunden. Die Alten Grundbücher wurden zwischen 1791 und 1794 von den Grundherrschaften angelegt und um 1880 von den bei den Bezirksgerichten geführten sogenannten Neuen handschriftlich geführten Grundbüchern abgelöst (vgl. Mayrhofer 2019). Auf Grundlage des Grundbuchumstellungsgesetzes von 1980 (BGBl. Nr. 550/1980) trat an ihre Stelle schließlich ein Datenbanksystem.

Das Hauptbuch des Neuen Grundbuches gliedert sich in Katastralgemeinden, innerhalb derer die Liegenschaften mit Einlagezahlen versehen sind. Jede Einlage untergliedert sich wieder in drei Teile: Auf dem A-Blatt (Gutsbestandsblatt) finden sich alle zur Liegenschaft gehörenden Grundstücke sowie die damit verbundenen Rechte, auf dem B-Blatt (Eigentumsblatt) sind die Eigentümer vermerkt und die Grundlage für den Eigentumserwerb wie Kauf oder Übergabe, auf dem C-Blatt (Lastenblatt) sind die Belastungen aufgelistet.

314 B.

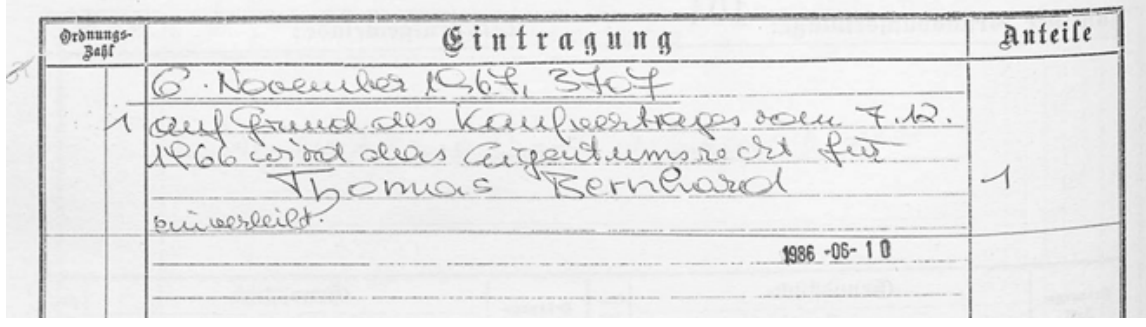

Abb. 5: Grundbucheintrag zum „Bernhard-Haus“ (B-Blatt). 


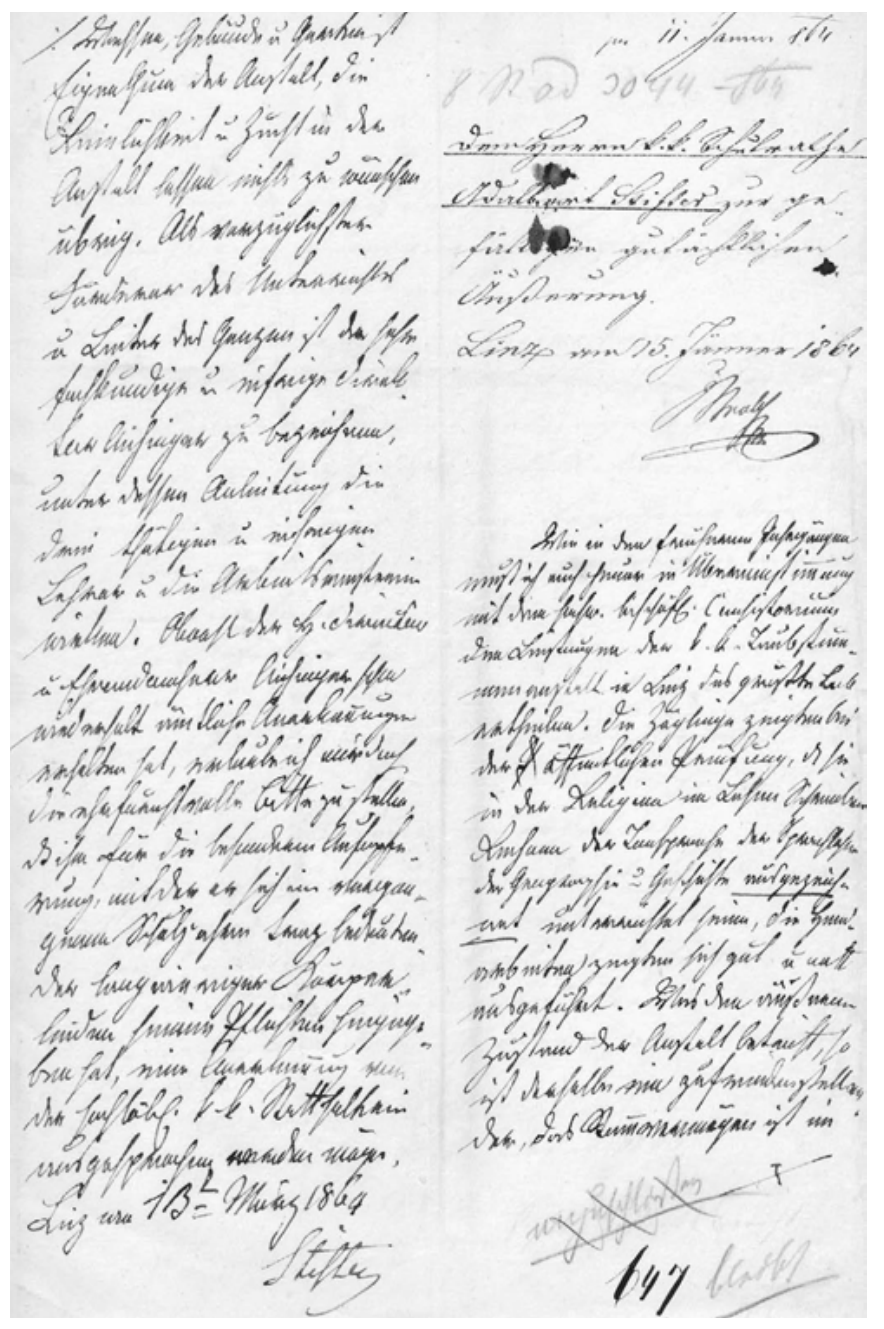

Abb. 6: Teil des Gutachtens von Adalbert Stifter zum Zustandsbericht Linzer Taubstummen Institut.

\section{Beruf}

Spuren in Archivalien kann auch berufliches Handeln hinterlassen, vor allem wenn man im Staatsdienst tätig ist, wie dies bei Adalbert Stifter als Schulrat der Fall war. Im OÖLA befindet sich, wie bereits erwähnt, der Bestand „Stifterakten“, der zum überwiegenden Teil aus Akten der k.k. Statthalterei besteht. Das bischöf- 


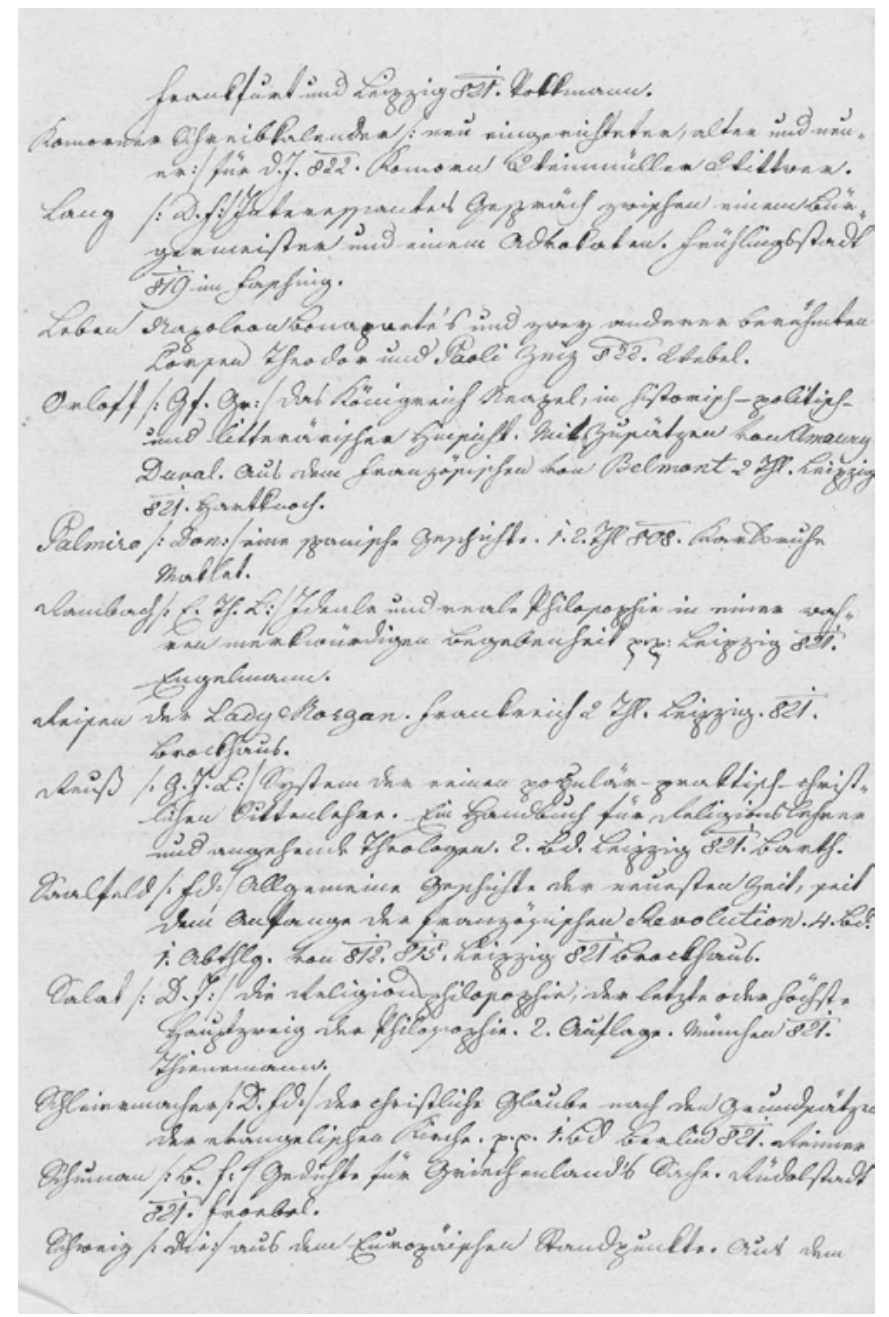

Abb. 7: „Verzeichnis der im Monat Dezember 1821 von der k.k. Central-Bücher-Zensur mit allerhöchster Genehmigung verbothenen Bücher“.

liche Konsistorium legte der k.k. Statthalterei 1864 einen Zustandsbericht zum Linzer Taubstummen Institut vor, zu dem Adalbert Stifter im März 1864 eine sehr positive Stellungnahme abgab und die k.k. Statthalterei ersuchte, dem Direktor des Instituts für seine Arbeit Anerkennung auszusprechen.

Texte als Ergebnis schriftstellerischer oder auch wissenschaftlicher Arbeit wurden immer wieder selbst zum Gegenstand staatlichen Handelns wie der repressiven Maßnahmen der Zensur im Metternich’schen Österreich. Ihre Spuren finden 
sich ebenfalls in den Verwaltungsakten des 19. Jahrhunderts, in diesem Fall im Bestand „Mühlkreisamt“. ${ }^{3}$ In einem Verzeichnis der Zentral-Bücher-Zensur finden sich bspw. das Buch der irischen Schriftstellerin Sydney Lady Morgan Reisen der Lady Morgan (1822) oder Friedrich Schleiermachers Der christliche Glaube nach den Grundsätzen der evangelischen Kirche (1830/31).

\section{Theater}

Im OÖLA befinden sich mehrere Bestände zum Themenbereich Theater. Dazu zählt die „Theaterzettelsammlung“ mit zum überwiegenden Teil Ankündigungen von Aufführungen des Ständischen Theaters in Linz aus dem 19. Jahrhundert. Ursprünglich Teil des Ständischen Archivs wurden die Theaterzettel in einer eigenen Sammlung zusammengezogen und um spätere Erwerbungen ergänzt.

Im Bestand „Landestheater“ finden sich Unterlagen zu Verwaltung, Engagements und Aufführungen beginnend in den 1940er-Jahren. Im September 1941 ersuchte das Landestheater bei der Reichskanzlei in Berlin um Refundierung der Kosten für die Neuausstattung der Operette Der Zigeunerbaron, die von Adolf Hitler bei Benno von Arent in Auftrag gegeben worden war. Ein umfangreicher Schriftverkehr im Vorfeld zeigt die Mühen rund um das Beschaffen von Bezugsscheinen für Materialien, von Leder bis Leinen, zur Herstellung der Ausstattung.

\section{Andenken und Ehrungen}

Niederschlag in Archivbeständen finden auch Ehrungen wie die Errichtung des Stifter-Denkmales an der Linzer Promenade. Der Denkmalausschuss wählte zur Umsetzung allerdings nicht den mit dem ersten Preis ausgezeichneten Entwurf, sondern den zweitgereihten von Johann Rathausky.

Das Ministerium für Cultus und Unterricht, das das Denkmal aufgrund zu geringen Spendenaufkommens mitfinanzierte, überreichte Landeshauptmann Dr. Alfred Ebenhoch eine Mappe mit den Concurrenz-Entwürfen für das StifterDenkmal. Das Denkmal wurde am 24. Mai 1902 feierlich enthüllt. Einer der gela-

3 Die Kreisämter gehen auf das Jahr 1749 zurück und wurden unter der Herrschaft von Maria Theresia geschaffen. Im Land ob der Enns umfassten sie jeweils ein Landesviertel (Hausruck-, Traun-, Machland- und Mühlkreisamt). Mit dem Erwerb des Innviertels 1779 wurden das Mühlund Machlandkreisamt zusammengelegt und in Ried zusätzlich ein Innkreisamt eingerichtet. 1850 traten die Bezirkshauptmannschaften als Mittelbehörden an ihre Stelle. 


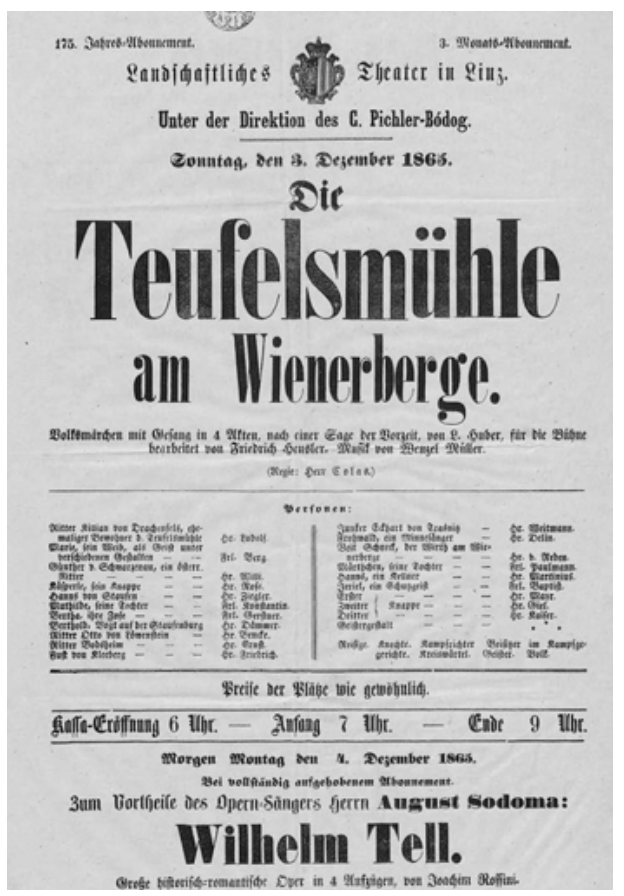

Abb. 8: Theaterzettel zur Aufführung von Die Teufelsmühle am Wienerberg, 3. Dezember 1865.

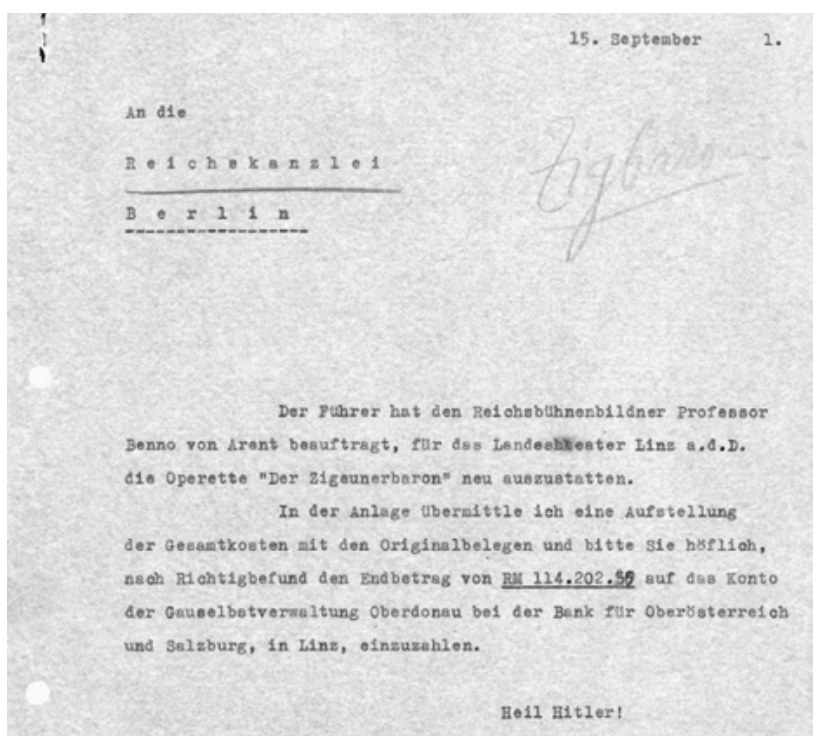

Abb. 9: Konzept eines Schreibens des Linzer Landestheaters an die Reichskanzlei. 


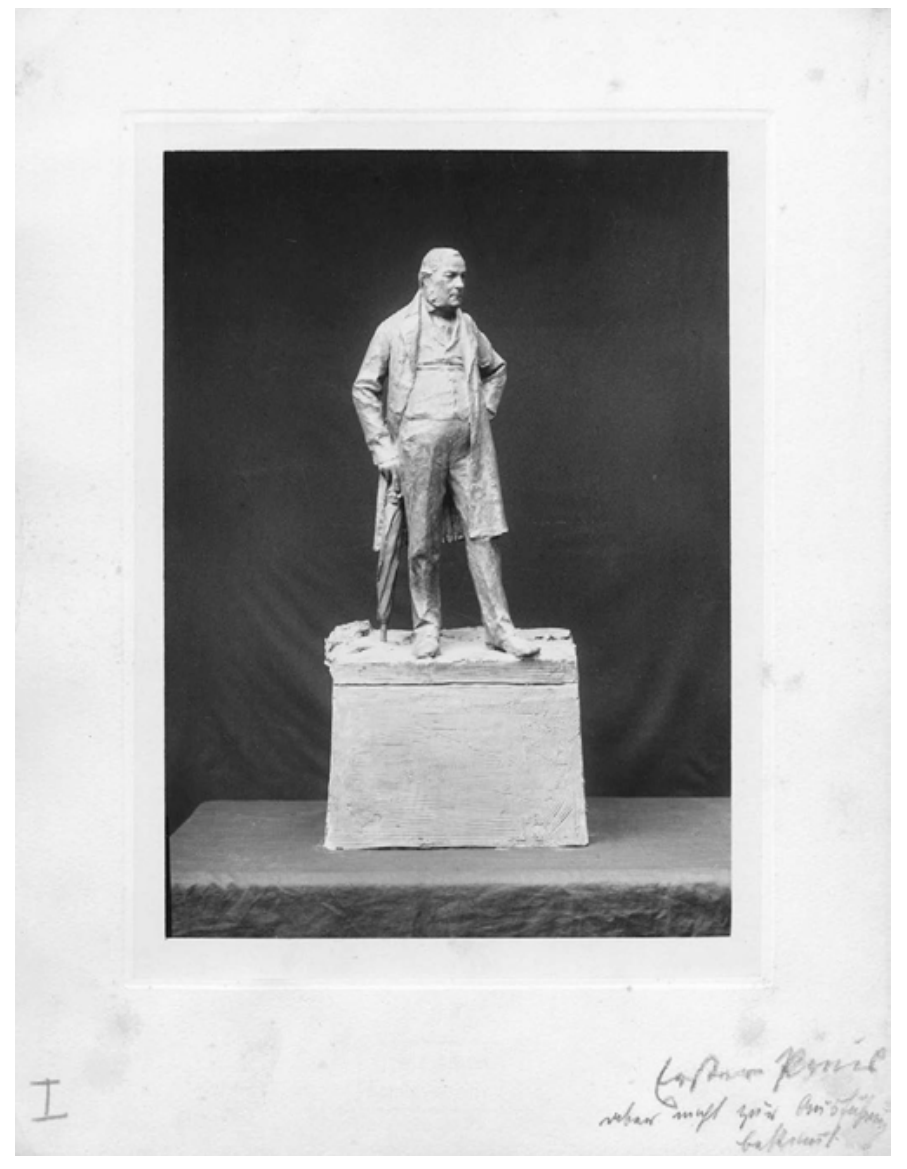

Abb. 10: Entwurf für das Stifter-Denkmal (erster Preis) von Othmar Schimkowitz; mit hs. Anmerkung: „aber nicht zur Ausführung bestimmt“.

denen Gäste, Hermann Bahr, konnte an der Feier nicht teilnehmen, da er in Salzburg bei der Beerdigung seiner Mutter war. In seiner Antwort auf die Einladung führte er aus, dass es u. a. Shakespeare, Goethe, Stifter und Stelzhamer sind, denen er seine „ganze geistige Existenz recht eigentlich verdanke“.

Dieser Brief hat sich gemeinsam mit weiteren Rechnungen, Korrespondenzstücken und Eingaben zum Stifter-Denkmal im Bestand „Musealarchiv“ erhalten. 1833 gründete Anton Ritter von Spaun den „Verein des vaterländischen Museums für Österreich ob der Enns mit Inbegriff des Herzogthums Salzburg“ und legte damit den Grundstein für das heutige Oberösterreichische Landesmuseum. Das neue Museum sammelte auch Archivalien unterschiedlichster Herkunft. Es finden sich darin Teile von Herrschaftsarchiven ebenso wie genealogische Unter- 

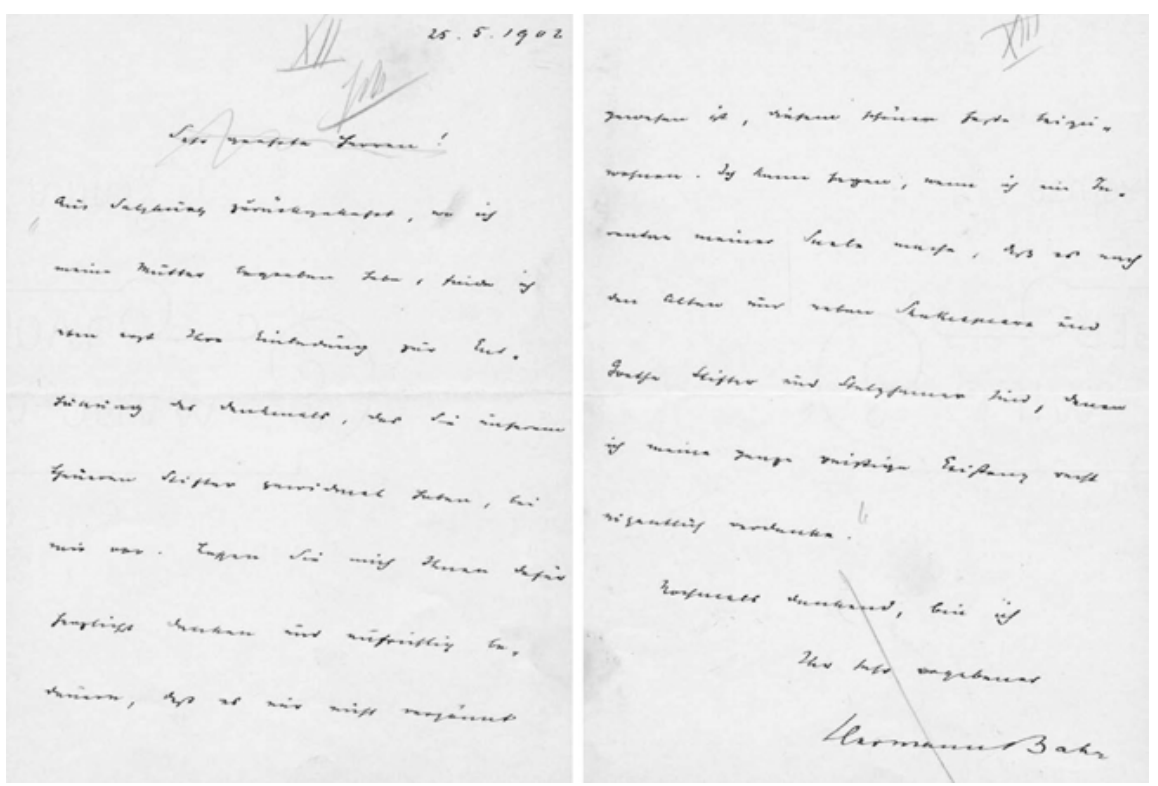

Abb. 11: Brief von Hermann Bahr an N. N., 25. Mai 1902.

lagen, kleinere Nachlässe und Material zu den Bauernkriegen. 1914 wurde der Bestand dem OÖLA übergeben.

\section{Fotografien}

Besonders anschauliche Quellen zu Personen oder Ereignissen sind natürlich Fotos. Auch wenn sie immer nur eine Momentaufnahme bieten, vermitteln sie vollkommen andere Eindrücke als schriftliche Quellen. In der Vierteljahresschrift des Adalbert-Stifter-Instituts findet sich ein kurzer Bericht über die Salzkammergut-Exkursion und den Aufenthalt in Bad Ischl, die im Rahmen der I. Internationalen Tagung des Adalbert-Stifter-Institutes im Mai 1955 anlässlich des Jubiläumsjahres zum 150. Geburtstag Stifters stattfand:

In Ischl wurden die Gäste im Hotel „Zur Post“ von Landeshauptmann Dr. Gleißner in Anwesenheit des Herrn Bürgermeister Franz Müllegger und des Oberlehrers Ramsauer empfangen. Kunisch machte sich zum Spruchsprecher der festfröhlich gestimmten Gesellschaft [...]. Anschließend führte Oberlehrer Ramsauer über den „Waldsteig“, der ein Stück StifterLandschaft in greifbarer Anschaulichkeit wachhält. (Adalbert-Stifter-Institut des Landes Oberösterreich 1956, 19-20) 
$\mathrm{Zu}$ dieser Salzkammergut-Exkursion hat sich in der Fotosammlung des OÖLA ein Bild erhalten.

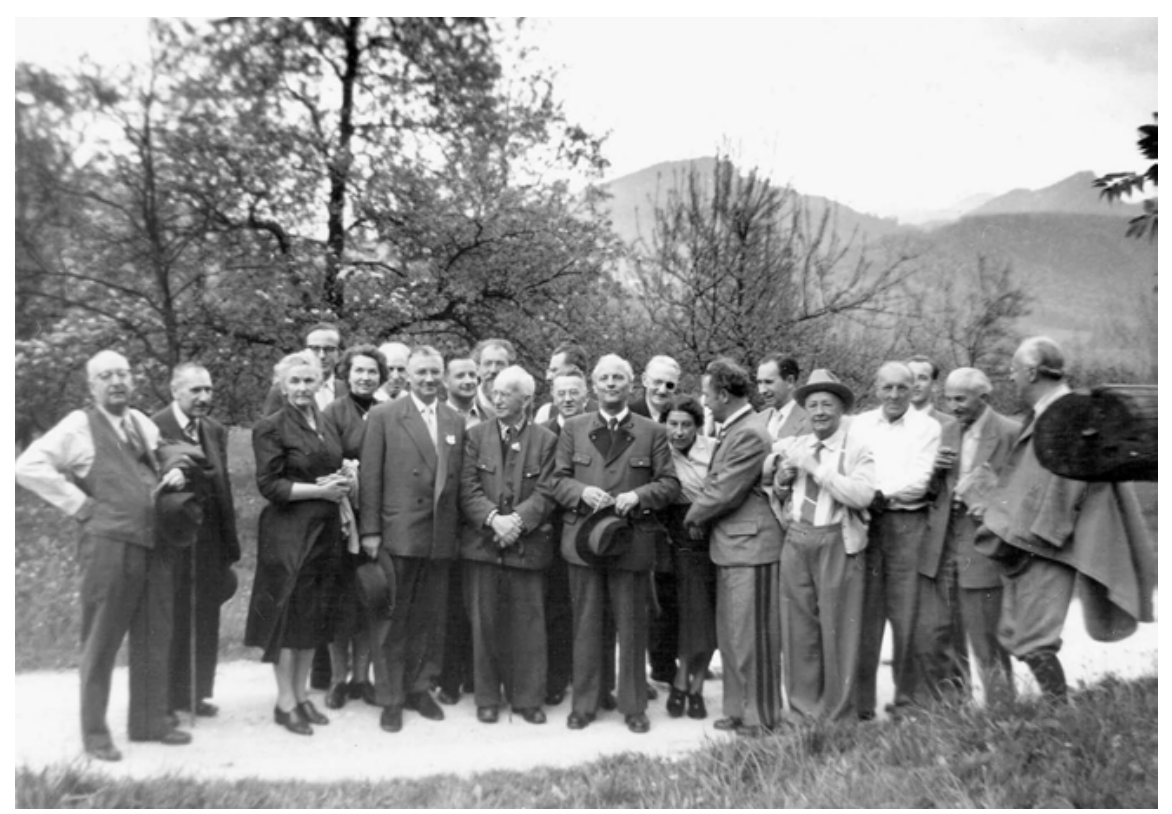

Abb. 12: Teilnehmerinnen und Teilnehmer der I. Internationalen Tagung des Adalbert-Stifter-Instituts mit Landeshauptmann Heinrich Gleißner in Bad Ischl.

\section{Resümee und Ausblick}

Wie gezeigt, beschränken sich die Bestände des OÖLA nicht nur auf die Dokumentation staatlichen Verwaltungshandelns in Oberösterreich, sondern bieten in ihrer Gesamtheit einen tiefen Einblick in die Geschichte des Landes. Im Gegensatz zur landläufigen Meinung bestehen die Aufgaben eines Archivars nicht nur im Erhalt des überlieferten Archivgutes, sondern vor allem auch in einer genauen historischen Bewertung und Feststellung der Archivwürdigkeit des heute entstehenden Schriftgutes, um künftigen Generationen ein Verständnis unserer Zeit zu ermöglichen.

Mit der zunehmenden digitalen Durchdringung des alltäglichen Lebens stehen die ArchivarInnen heute vor einer Herausforderung, mit der frühere Archivarsgenerationen in diesem Ausmaß nie konfrontiert waren: der kompletten Änderung des Verhältnisses von Information zu Informationsträger. Information, 
beispielsweise Schrift, war bisher meist mit ihrem Informationsträger, beispielsweise Papier, fest verbunden. Sorgte man für den Erhalt des Informationsträgers, war auch die Information erhalten. Im Bereich der digitalen Information würde so eine Vorgehensweise zu einem Totalverlust führen. Der Erhalt des Informationsträgers, beispielsweise einer 8-Zoll-Diskette, hätte zur Folge, dass die darauf gespeicherten Daten heute entweder gar nicht mehr abspielbar wären oder nur mit großem technischem Aufwand rekonstruiert werden könnten. Bei der digitalen Archivierung steht daher der Erhalt der Information im Vordergrund, der Informationsträger hat höchstens musealen Wert. Die Information muss allerdings nicht „nur“ erhalten werden, sondern ihre Authentizität und Integrität muss auch nach Jahrzehnten und möglicherweise mehrfachen Migrationen gewährleistet bleiben.

Digitale Information führt außerdem zu einer massiven Beschleunigung im Bereich Archivierung. Die berühmten Aktenfunde in vergessenen Kellern oder die verstaubten Tagebücher der Großeltern auf Dachböden wird es künftig nicht mehr geben. Um unsere digitale Gegenwart zu erhalten, muss Archivierung viel früher einsetzen als bisher, da digitale Information wesentlich flüchtiger ist als beispielsweise Niedergeschriebenes. Gerade dieser Umstand macht Archivierung heute zu einem hochspannenden Thema. Womit sich der Kreis wieder schließt: Archive sind zwar meist ,altehrwürdige“ Institutionen, allerdings mit einem sehr innovativen Innenleben. Anders könnten die Herausforderungen der Archivierung unserer Zeit nicht gelöst werden.

\section{Literaturverzeichnis}

Adalbert-Stifter-Institut des Landes Oberösterreich. „Das Jubiläumsjahr 1955“. Vierteljahresschrift des Adalbert-Stifter-Institutes des Landes Oberösterreich 5 (1956), F. 1/2: 6-26.

BGBI. Nr. 264/1951. Geschäftsordnung für die Gerichte I. und II. Instanz (Geo.). https://www.ris. bka.gv.at/GeltendeFassung.wxe?Abfrage=Bundesnormen\&Gesetzesnummer $=10000240$ (22.1.2020).

BGBI. Nr. 550/1980. Bundesgesetz vom 27. November 1980 über die Umstellung des Grundbuchs auf automationsunterstützte Datenverarbeitung und die Änderung des Grundbuchsgesetzes und des Gerichtskommissärsgesetzes (Grundbuchsumstellungsgesetz-GUG). https://www.ris.bka.gv.at/GeltendeFassung.wxe?Abfrage= Bundesnormen\&Gesetzesnummer=10002501 (22.1.2020).

BGBI. I Nr. 16/2013. Bundesgesetz, mit dem das Bundesgesetz über die Regelung des Personenstandswesens (Personenstandsgesetz 2013 - PStG 2013) erlassen sowie das Staatsbürgerschaftsgesetz 1985, das Meldegesetz 1991 und das Namensänderungsgesetz geändert werden und das Personenstandsgesetz aufgehoben wird. https://www.ris.bka. gv.at/eli/bgbl/I/2013/16 (22.1.2020). 
Haider, Siegfried. „100 Jahre Oberösterreichisches Landesarchiv. Das älteste wissenschaftliche Landesinstitut (1896-1996)“. Landesgeschichte und Archivwissenschaft. Festschrift zum 100jährigen Bestehen des OÖ. Landesarchivs. Hg. Oö. Landesarchiv. Linz: OÖLA, 1996. $5-36$.

LGBI. Nr. 83/2003. Landesgesetz über die Sicherung, die Aufbewahrung und die Nutzung von öffentlichem Archivgut sowie die Tätigkeit der damit betrauten Archive (Oö. Archivgesetz). https://www.ris.bka.gv.at/GeltendeFassung.wxe?Abfrage= LrOO\&Gesetzesnummer=20000258 (22.1.2020).

Mayrhofer, Willibald. Quellenerläuterungen für Haus- und Familienforschung in Oberösterreich. 5. überarb. u. erw. Aufl. Linz: Oö. Landesarchiv, 2019. 